\title{
Hepatic Angiomyolipoma: Case Report, Literary Review with Emphasis on Imaging Performance for the Diagnosis
}

\author{
Gil-Serrano PE1, Daza-Cajas $\mathrm{G}^{2}$, Chaustre-Soledad $\mathrm{JE}^{3 *}$ and \\ Vasquez $\mathrm{AY}^{4}$ \\ ${ }^{1}$ Department of Radiology, Universidad CES Cali, Colombia \\ ${ }^{2}$ Assistant Professor Radiology Department, Universidad del Valle Cali, Colombia \\ ${ }^{3}$ Universidad De La Sabana, Colombia \\ ${ }^{4}$ Radiology Resident Physician, Universidad De La Sabana, Colombia
}

\section{Case Report \\ Volume 3 Issue 4}

Received Date: November 12, 2019

Published Date: November 29, 2019

DOI: $10.23880 /$ crij-16000158

*Corresponding author: Chaustre-Soledad JE, Universidad De La Sabana, Bogotá, Colombia; Email: jose.chaustre@hotmail.com

\section{Abstract}

Angiomyolipoma is a rare mesenchymal neoplasm that is composed of: blood vessels, smooth muscle and fat cells. The kidneys are the site where they can be most commonly located, while the liver is a less common site of origin; these hepatic lesions are often discovered as an incidental finding in the search for diagnostic images for another pathology or are identified in the evaluation of non-specific symptoms. This tumor has been defined as a benign neoplasm, although it is suggested that some cases may have a malignant potential after hepatic resection. The definitive diagnosis is based on pathological findings supported by immunohistochemical staining. The cellular component of smooth muscle is the most specific for the diagnosis and, characteristically, these lesions are positive for homatropine methyl bromide 45 (HMB-45) and Melan-A. Because hepatic angiomyolipomas are rare and have several imaging characteristics that tend to be confused with other tumors due to their very similarity, it is not easy to achieve a definitive preoperative diagnosis, and more than half of patients are misdiagnosed with hepatocellular carcinoma or hepatic adenoma, which are much more common lesions. Regarding the imaging diagnosis of angiomyolipoma; the fatty component can be detected by CT and ultrasound. When the fat content is lower, the magnetic resonance images in weighted and diffusion sequences are useful due to their sensitivity. In fact, magnetic resonance using conventional sequences and weighted by diffusion have become a valuable technique for the characterization of focal solid liver lesions.

Keywords: Hepatic Angiomyolipoma; Benign Neoplasm; Computed Tomography; Hepatocellular Carcinoma; Magnetic Resonance Imaging 


\section{Clinical Radiology \& Imaging Journal}

\section{Clinical Case}

53-year-old female patient, with no significant pathological history; who presents an 8-month clinical picture of abdominal pain and bloating, without other associated symptoms. In the initial assessment, abdominal ultrasound was performed in which nodular, solid, echogenic image located in segment II of the liver was evidenced, at the color Doppler assessment there was no flow; with this finding, a contrasting tomography of the abdomen is performed, evidencing that the lesion described in ultrasound is hypodense and presents late enhancement after the administration of the intravenous contrast medium; although its enhancement is not complete. On magnetic resonance imaging, the liver lesion is hyperintense in the T2 enhanced sequences, in the T1 sequences it is slightly hyperintense; in the out-of-phase sequences and in the $\mathrm{T} 2$ sequences with fat saturation (fat sat), the signal falls. The patient was diagnosed with a hepatic AML and no biopsy wasn't considered because, the patient rejected invasive procedures. The patient continues in controls by external consultation, and until now, 2 years after the intervention, she has evolved adequately, has not presented any symptoms, or signs of recurrence, additionally the imaging controls do not report growth of the lesion, In the present case, the patient did not agree to perform any type of invasive diagnostic procedure, which is why periodic imaging controls are performed, which so far, 24 months later, have not shown changes with respect to the initial images.

\section{Figure Findings}

\section{Magnetic Resonance}

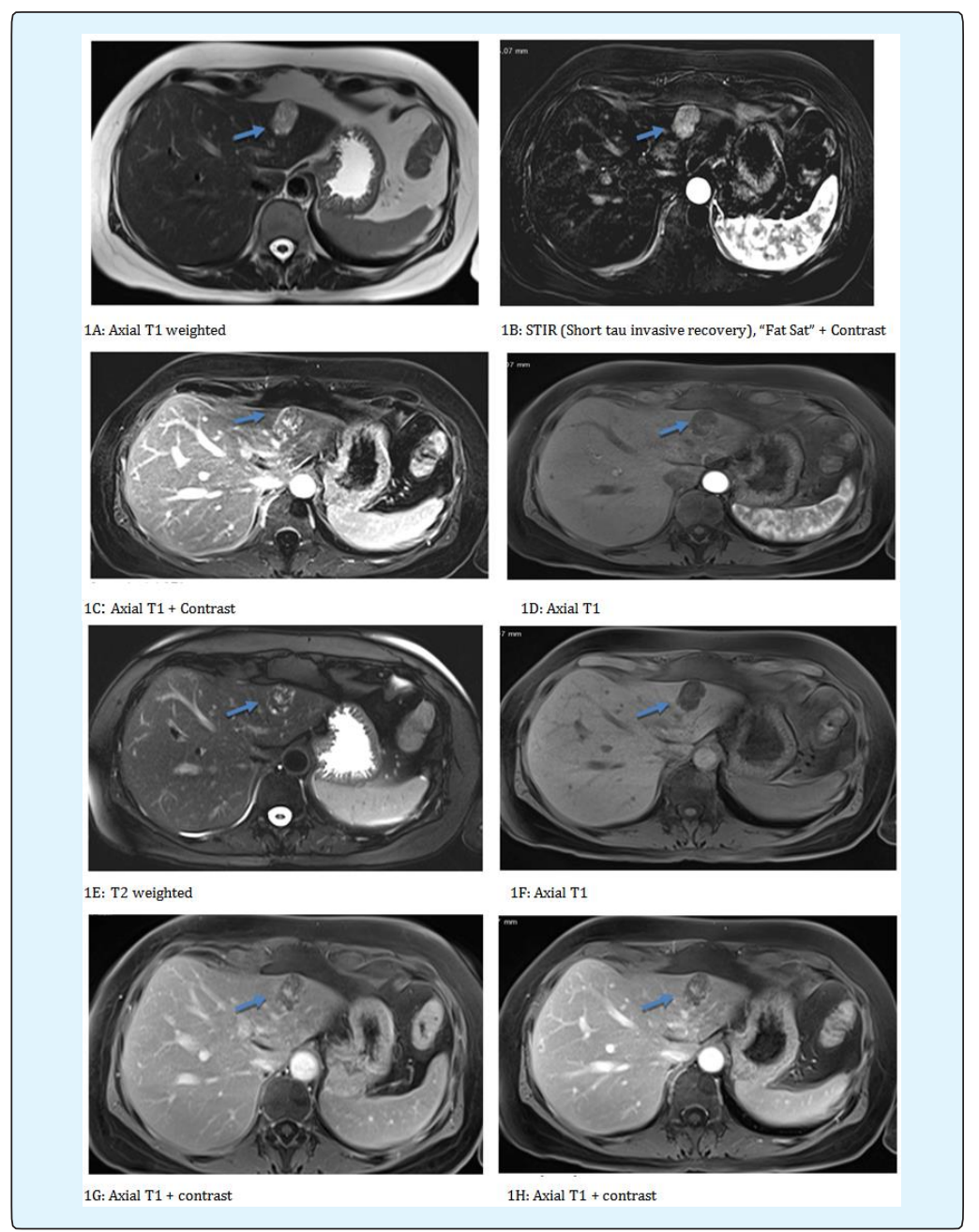

Chaustre-Soledad JE, et al. Hepatic Angiomyolipoma: Case Report, Literary Review with Emphasis on Imaging Performance for the Copyright@ Chaustre-Soledad JE, et al. Diagnosis. Clin Radiol Imaging J 2019, 3(4): 000158. 


\section{Clinical Radiology \& Imaging Journal}

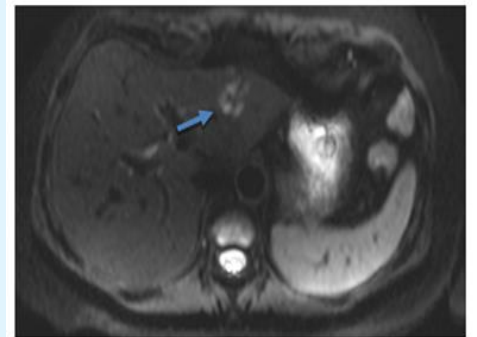

1I: Diffusion weighted

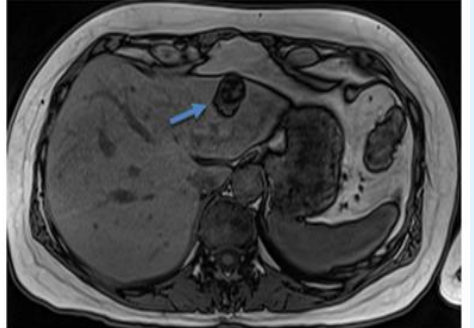

1): Axial T1, out of phase

Figure 1: MR-In the left hepatic lobe on the II segment, a $2.9 \times 2.3 \mathrm{~cm}$ diameter hyperintense focal lesion is found in T1, T2, (Figures 1A-1E) and hypointense in T2 Fat sat (Figure 1B). With significant signal strength decay in opposite phase sequences and VIBE T1 sequences (Figures 1F-1J).

Indicating macroscopic fatty content inside which heterogeneously enhances after the administration of contrast without a specific pattern and that in the late stages does not fill completely. In addition, there is no rapid washing at the lesion enhancement. Findings suggestive of hepatic amgiomyolipoma. It is important to highlight in sequences with fat suppression (Figure 1B): drop in signal intensity due to the high lipid content.

\section{CT Scan}

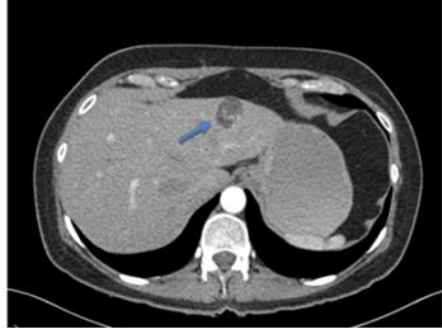

2A: Axial CT + contrast

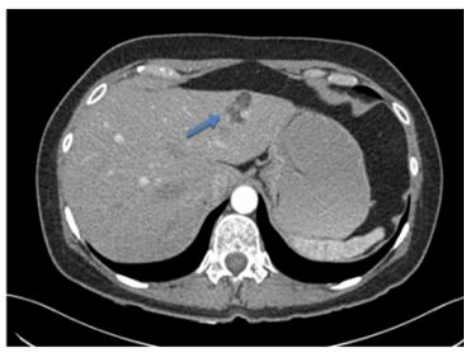

2c. Axial CT + contrast

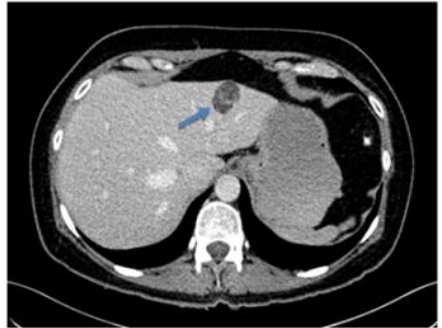

2B: Axial CT without contrast

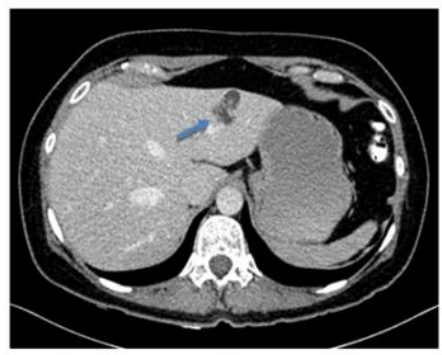

2D: Axial CT + contrast (late arterial phase)

Figure 2: CT scan- Computed tomography of ambdomen in simple phase Axial cut, a solid-looking image is identified, with heterogeneous attenuation values due to the presence of fat and soft tissue densities; predominantly hypodense, located in the segment II. 


\section{Clinical Radiology \& Imaging Journal}

\section{Ultrasound}

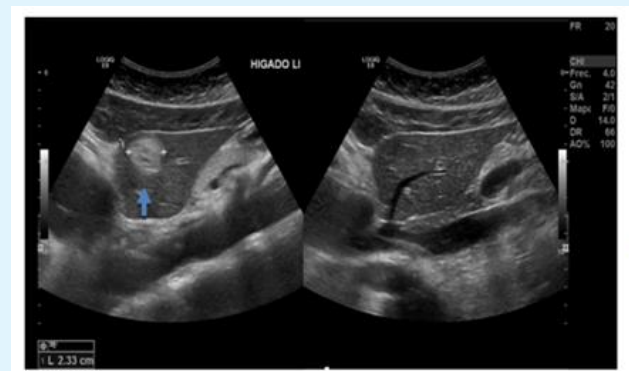

3A: Gray Scale

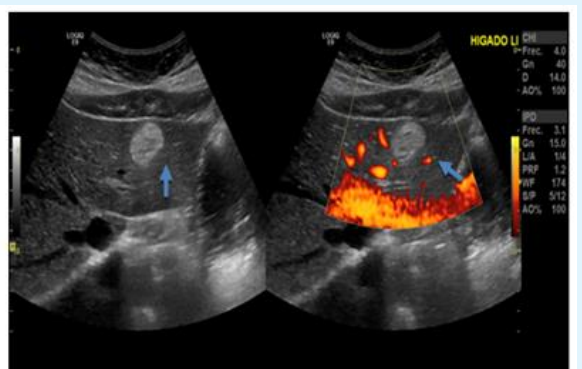

3B: Gay scale + Doppler

Figure 3: Ultrasound- Image of nodular appearance, solid, ecogenic (due to fat content) or heterogeneous echogenicity in the segment II of the liver (blue arrow). In case the lesion has low lipid content and high vascular content, the angiomyolipoma may be hypoechoic. At color doppler titration, there is an increase in vascular flow (3B).

\section{Discussion}

Hepatic angiomyolipoma is a rare benign mesenchymal neoplasm, first described in 1976 by Ishak $\mathrm{KG}$ and his team [1], it is a much more common pathology in women, its etiology has not yet been fully elucidated Yeh CN, et al. [2]. Despite the advanced imaging techniques currently available, diagnosis is still difficult preoperatively, since the lesions are made up of a mixture of cells, including smooth muscle cells, fat cells and blood vessels that occur in varying proportions in different locations of a single lesion generating image patterns that resemble a wide range of differential diagnoses [3]. In a study of 178 patients published in 2013 by Yang X, et al. 41 (23\%) presented symptoms, which included abdominal discomfort, weight loss, fever, nausea, diarrhea, asthenia-adinamia and mass sensation due to progressive enlargement of the tumor [4]. The fact that the majority of patients do not present clinical and also, the percentage of patients who report any symptoms report really nonspecific symptoms that make it not easy to type the angiomyolipoma as a diagnostic probability. In this review we emphasize the different possibilities of diagnostic imaging.

\section{Ultrasonography}

In a cohort of 178 patients undergoing surgery for angiomyolithoma, all patients underwent an ultrasound examination and only two or more lesions were found in 13 of the patients [4]. Regarding the findings; the lesion can be highly echogenic and, therefore, cannot be distinguished from a hemangioma. When present, sound attenuation, velocity propagation device and refractive device allow distinguishing characteristic angiomyolipoma lesions from other echogenic tumors, especially hemangiomas. However, an image of greater sensitivity will always be necessary to properly characterize the lesion, so the ultrasound works in the context of an initial evaluation but not as a definitive diagnostic method [5-8].

\section{Computed Tomography}

As for tomography, it has been shown that angiomyolipoma consists of two parts: a peripheral angiomyomatous component with soft tissue attenuation and a fatty component with an attenuation value of less than $20 \mathrm{UH}[5,4,9]$. Also, the lesions often contain a part of a hemangioma-like component within the tumor. In a study published in 2015 by Kuramoto K, et al. [5], three of the four patients presented in this study demonstrated hypervascular nodules, early venous filling of the contrast medium and no visible direct shunts or major vascular abnormalities were observed [8]. In addition, the phenomena of early vein enhancement could be useful in differentiation with the other liver tumors and also caused a delayed washing in the arterial component, similar to hemangioma. This makes the tomography a study with much higher performance since it gives us more information to generate and discard differential diagnoses properly [5,10-12].

\section{Magnetic Resonance}

The characteristics of the resonance vary depending on the proportion of intra-tumor fat and clearly offer better performance compared to the aforementioned modalities [7,9,13]. Frequently, angiomyolipomas have a high fat content, with high signal intensity in T1-weighted 


\section{Clinical Radiology \& Imaging Journal}

images and a significant decrease in signal intensity in images with fat suppression [14]. However, according to Balci NC, et al. tumors with a substantial fatty content tend to present a diagnostic challenge since they can be easily confused with any other focal liver lesion [13]. The diffusion-weighted images are sensitive to molecular diffusion and allow tissue characterization by being able to identify the microstructural changes of the tissue in much smaller lesions. Therefore, diffusion images in addition to the images obtained from a routine resonance would increase the diagnostic performance of radiologists during the evaluation of focal liver lesions [7,9]. It should also be taken into account that high signal intensity is an expression of different pathological changes: fat deposition, copper accumulation, high protein concentration, blood degradation and sinusoidal dilation. It can also be observed in malignant liver tumors such as Hepatocarcinoma and metastases of primary tumors such as melanoma $[12,15]$.

\begin{tabular}{|c|c|c|}
\hline Type of study & Correct diagnosis & Incorrect / inconclusive diagnosis \\
\hline US & 1 & 177 \\
\hline TC & 13 & 102 \\
\hline RM & 7 & 74 \\
\hline
\end{tabular}

Table 1: Preoperative diagnostic performance of three modality images in a study of 178 patients undergoing surgery for hepatic angiomyolipoma. The yield for each of the images was $11 \%$ CT, $8.6 \%$ RM and $0.56 \%$ US. Being, in this study the tomography the image with greater performance. However, we consider that the resonance is the image that provides the most data when characterizing the lesions [4].

\section{Pathological and Immunohistochemical Study}

The pathological study of hepatic angiomyolipoma generally shows a unique pattern, well circumscribed and not encapsulated, which tends to consist of connective and elastic tissue. The cutting surfaces of the tumors were brown-yellowish, some of them showed centers of necrosis, areas of hemorrhage and cystic degeneration. In microscopic evaluation, a mixture of blood vessels, epithelial cells or even smooth muscle and mature adipose tissue is usually evident $[7,14]$. A large number of thin-walled venous vascular structures or blood sinuses that converge in the tumor parenchyma can be found [14]. In a case series of 94 patients carried out by Chang Z, et al. [14] it was shown that hematopoietic elements and infiltration of inflammatory cells were often present within the tumors. In addition, they evidenced the tumor tissues occasionally presented an obvious invasion. In the surrounding liver tissue with an infiltrating edge; however, no invasion of surrounding blood vessels was found. When a hypervascular liver lesion containing an atypical fatty component is found, immunohistochemistry with markers of hepatocyte (anti-hepatocyte), adipocyte (PS100) and PEComas (HMB45, Melan A, AML) is essential to avoid diagnostic errors [16].

\section{Conclusion}

Hepatic angiomyolipoma is a rare and rare neoplasm whose diagnosis presents an important update when it comes to differentiating the imaging findings and which can present important variations in the different imaging methods. The findings can be evaluated by ultrasonography, computed tomography and magnetic resonance, the latter being the image that shows the highest performance. The fatty component should always search in T1 weighted sequences, it is important to use sequences with fat saturation to better differentiate said component. Histopathologically, a unique pattern, well circumscribed and not encapsulated, consisting of a connective and elastic tissue is normally shown. It is basically composed of blood vessels, smooth muscle and fat cells. We present a case of a 53-year-old patient, with no significant pathological history; who presents an 8month clinical picture of abdominal pain and bloating, without other associated symptoms. In this patient a purely radiological diagnosis was made and imaging was done for 24 months without finding changes in the observed images. It could be considered as a diagnostic method the use of multiple radiological images that, as in this case, match procedures with the typical characteristics of liver angiomyolipoma in case of contraindicating invasive procedures, as in our case as the patient refused it.

\section{References}

1. Ishak KG Mesenchymal tumors of the liver. Hepatocell carcinoma, In: Okuda K and Peters RL (Eds.), pp: 247307.

2. Yeh CN, Lee KF, Chen MF (2005) Immunohistochemical study of hepatic 


\section{Clinical Radiology \& Imaging Journal}

angiomyolipoma. Hepatogastroenterology 52(64): 1151-1153.

3. Murakami T, Tsurusaki M (2014) Hypervascular Benign and Malignant Liver Tumors That Require Differentiation from Hepatocellular Carcinoma: Key Points of Imaging Diagnosis. Liver Cancer 3(2): 85-96.

4. Yang X, Li A, Wu M (2013) Hepatic angiomyolipoma: clinical, imaging and pathological features in 178 cases. Med Oncol 30(1): 416.

5. Kuramoto K, Beppu T, Namimoto T, Hayashi H, Imai $\mathrm{K}$, et al. (2015) Hepatic angiomyolipoma with special attention to radiologic imaging. Surg Case Rep 1(1): 38.

6. Wang CP, Li HY, Wang H, Guo XD, Liu CC, et al. (2014) Hepatic Angiomyolipoma Mimicking Hepatocellular Carcinoma: magnetic resonance imaging and clinical pathological characteristics in 9 cases. Medicine 93(28): 194.

7. Wei H, Liu H, Chen Y, Xue X, Weng H, et al. (2017) The Histopathological Features and CT/MRI Imaging Performances in Hepatic Angiomyolipoma Patients. Ann Hepatol 16(5): 759-764.

8. Prasad SR, Wang H, Rosas H, Menias CO, Narra VR, et al. (2005) Fat-containing Lesions of the Liver: Radiologic-pathologic correlation. Radiographics 25(2): 321-331.

9. Wang SY, Kuai XP, Meng XX, Jia NY, Dong H (2014) Comparison of MRI features for the differentiation of hepatic angiomyolipoma from fat-containing hepatocellular carcinoma. Abdom Imaging 39(2): 323-333.
10. Nguyen TT, Gorman B, Shields D, Goodman Z (2008) Malignant Hepatic Angiomyolipoma: Report of a Case and Review of Literature. Am J Surg Pathol 32(5): 793-798.

11. Zeng JP, Dong JH, Zhang WZ, Wang J, Pang XP (2010) Hepatic Angiomyolipoma: A Clinical Experience in Diagnosis and Treatment. Dig Dis Sci 55(11): 32353240 .

12. Yoshimura $H$, Murakami T, Kim T, Nakamura $H$, Inoue $Y$, et al. (2002) Angiomyolipoma of the liver with least amount of fat component: imaging features of CT, MR, and angiography. Abdom Imaging 27(2): 184-187.

13. Balci NC, Akinci A, Akun E, Tunaci A (2002) Hepatic angiomyolipoma: demonstration by out of phase MRI. Clin Imaging 26(6): 418-420.

14. Chang Z, Zhang JM, Ying JQ, Ge YP (2011) Characteristics and treatment strategy of Hepatic angiomyolipoma: a series of 94 patients collected from four institutions. J Gastrointestin Liver Dis 20(1): 65-69.

15. Takayama Y, Moriura S, Nagata J, Hirano A, Ishiguro S, et al. (2002) Hepatic angiomyolipoma: radiologic and histopathologic correlation. Abdom Imaging 27(2): 180-183.

16. Lafitte M, Ayav A, Bressenot A, Claudon M, Regent D, et al. (2013) Radiologic-pathologic correlation in liver angiomyolipoma in a 68-year-old woman. Diagn Interv Imaging 94(11): 1161-1164. 